\title{
A COMPARATIVE STUDY OF THE ZEROS OF DIRICHLET $L$-FUNCTIONS
}

\author{
BY AKIO FUJII ${ }^{1}$ \\ Communicated by Paul Bateman, June 18, 1974
}

We give a comparative study of the zeros of Dirichlet $L$-functions.

Details will appear later.

1. Let $\chi_{1}$ and $\chi_{2}$ be distinct primitive characters of the same modulus $q$, and let $L\left(s, \chi_{i}\right)$, for $i=1,2$, be the corresponding Dirichlet $L$-functions. It is quite natural to guess that $L\left(s, \chi_{1}\right)$ and $L\left(s, \chi_{2}\right)$ have no coincident zero. In other words even a single zero will determine a Dirichlet $L$-function, or more generally, a "zeta-function". To be more precise, we call $\rho$ a coincident zero of $L\left(s, \chi_{1}\right)$ and $L\left(s, \chi_{2}\right)$ if $L\left(\rho, \chi_{1}\right)=L\left(\rho, \chi_{2}\right)=0$ with the same multiplicities. And we call $\rho$ a noncoincident zero if it is not coincident. Then we can show

THEOREM 1. Let $\chi_{1}$ and $\chi_{2}$ be distinct primitive characters of the same modulus. Then a positive proportion of the zeros of $L\left(s, \chi_{1}\right)$ and $L\left(s, \chi_{2}\right)$ are noncoincident.

Next, it is quite natural to guess that the distribution of the zeros of $L\left(s, \chi_{1}\right)$ and $L\left(s, \chi_{2}\right)$ are independent. To state our results, let $\gamma_{n}(\chi)$ be the ordinate of the $n$th zero of $L(s, \chi)$ such that $0 \leqslant \gamma_{n}(\chi) \leqslant \gamma_{n+1}(\chi)$. Further we define $\gamma_{n}\left(\chi_{1}\right) \leqslant \gamma_{m}\left(\chi_{2}\right)$ if $\gamma_{n}\left(\chi_{1}\right)<\gamma_{m}\left(\chi_{2}\right)$, and $\gamma_{n}\left(\chi_{1}\right) \leqslant$ $\gamma_{m}\left(\chi_{2}\right) \leqslant \gamma_{n+1}\left(\chi_{1}\right) \leqslant \gamma_{m+1}\left(\chi_{2}\right) \leqslant \cdots \quad$ if $\gamma_{n}\left(\chi_{1}\right)=\gamma_{n+1}\left(\chi_{1}\right)=\cdots=$ $\gamma_{m}\left(\chi_{2}\right)=\gamma_{m+1}\left(\chi_{2}\right)=\cdots$. Then we get

THEOREM 2. Under the same hypothesis as above, for a positive proportion of $\gamma_{n}\left(\chi_{1}\right)$ 's, there does not exist a $\gamma\left(\chi_{2}\right)$ for which $\gamma_{n}\left(\chi_{1}\right) \leqslant \gamma\left(\chi_{2}\right) \leqslant$ $\gamma_{n+1}\left(\chi_{1}\right)$.

Further we define $\Delta_{n}\left(\chi_{1}, \chi_{2}\right)$ to be $n-m$ if $\gamma_{m}\left(\chi_{1}\right) \leqslant \gamma_{n}\left(\chi_{2}\right) \leqslant$

AMS (MOS) subject classifications (1970). Primary $10 \mathrm{H} 05,10 \mathrm{H} 10$.

Key words and phrases. Riemann zeta function, distribution of zeros.

${ }^{1}$ Supported in part by National Science Foundation grant GP 36418X1. 
$\gamma_{m+1}\left(\chi_{1}\right)$. Then we can show

THEOREM 3. For any positive increasing function $\Phi(n)$ which tends to $\infty$ as $n$ tends to $\infty$, we have

$$
\left|\Delta_{n}\left(\chi_{1}, \chi_{2}\right)\right|>2 \pi(\log \log n)^{1 / 2} / \Phi(n)
$$

for almost all $n$. In particular, $\gamma_{n}\left(\chi_{2}\right)$ almost never satisfies $\gamma_{n}\left(\chi_{1}\right) \leqslant$ $\gamma_{n}\left(\chi_{2}\right) \leqslant \gamma_{n+1}\left(\chi_{1}\right)$.

Theorems 1 and 2 come from a mean value theorem about

$$
\int_{0}^{T}\left\{S\left(t+h, \chi_{1}\right)-S\left(t, \chi_{1}\right)-\left(S\left(t+h, \chi_{2}\right)-S\left(t, \chi_{2}\right)\right)\right\}^{l} d t,
$$

where $S(t, \chi)=\pi^{-1} \arg L(1 / 2+i t, \chi)$ as before (cf. [1]). Theorem 3 comes from a mean value theorem about $\int_{0}^{T}\left(S\left(t, \chi_{1}\right)-S\left(t, \chi_{2}\right)\right)^{l} d t$. If we use mean value theorems about

$$
\sum_{\chi_{1}}^{\prime} \sum_{\chi_{2}}^{\prime}\left\{S\left(t+h, \chi_{1}\right)-S\left(t, \chi_{1}\right)-\left(S\left(t+h, \chi_{2}\right)-S\left(t, \chi_{2}\right)\right)\right\}^{l}
$$

and

$$
\sum_{\chi_{1}}^{\prime} \sum_{x_{2}}^{\prime}\left\{S\left(t, \chi_{1}\right)-S\left(t, \chi_{2}\right)\right\}^{l}
$$

where in the summation $\chi_{i}$ runs over all nonprincipal characters of modulus $q$ for each $i=1,2$, then we get $q$-analogues of our theorems.

2. As an application of our methods we can get some results about a problem of Knapowski-Turán. Let $q$ be a given fixed positive integer. Assume that $(b, q)=(d, q)=1$ and $b \not \equiv d(\bmod q)$. Let $\chi$ be a character of modulus $q$. We write $g(\chi)=(\bar{\chi}(b)-\bar{\chi}(d)) / \varphi(q)$, and $\mu(\rho)=\mu_{b, d}(\rho)=$ $\Sigma_{\chi} g(\chi) m_{\chi}(\rho)$, where $\chi$ runs over all characters of modulus $q$ and $m_{\chi}(\rho)$ is the multiplicity of $\rho$ as a zero of the Dirichlet $L$-functions $L(s, \chi)$. Knapowski and Turán proposed the following problem in their study of prime numbers:

Estimate $f(T)=\Sigma_{0<\operatorname{Im} \rho<T ; \mu(\rho) \neq 0} 1$ (cf. [3]). Concerning this problem, Kátai (unpublished) and Grosswald [2] proved independently the existence of infinitely many $\rho$ 's with $\mu(\rho) \neq 0$. Later Turán obtained the following results (cf. [6]).

(1) For $T>\psi(q)$ we have the inequality $f(T)>c_{1} \exp \left((\log T)^{1 / 5}\right)$. 
(2) Under the assumption of the generalized Riemann hypothesis we have $f(T)>C_{2} T^{1 / 2}$ for $T>\psi(q)$, where the $C_{\nu}$ are numerical constants and $\psi(q)$ is an explicit function of $q$. Recently Motohashi [4] obtained the following results.

(1) For $T>\psi(q)$ we have $f(T)>T^{1 / 10}(\log T)^{-3}$.

(2) For any sufficiently large $T$ there exists at least one $q$ with $1 / 2 T^{1 / 2}(\log T)^{-51} \leqslant q \leqslant T^{1 / 2}(\log T)^{-51}$ such that $f(T)>T^{3 / 28}(\log T)^{-45}$.

Now we can show

THEOREM 4. For $T>\psi(q)$ we have $f(T)>A T \log T$, where $\psi(q)$ is some explicit function of $q$ and the positive constant $A$ may depend on $q$.

In fact, we can take $\psi(q)=\exp \left(\exp \left(C_{1} q\right)\right)$ and $A=\exp \left(-C_{2} q\right)$ with suitable positive absolute constants $C_{1}$ and $C_{2}$.

We prove this from a mean value theorem concerning

$$
\int_{0}^{T}\left|\sum_{\chi} g(\chi)\left(S\left(t+h, \chi^{*}\right)-S\left(t, \chi^{*}\right)\right)\right|{ }^{l} d t,
$$

where $\chi^{*}$ is the primitive character attached to $\chi$.

\section{REFERENCES}

1. A. Fujii, On the distribution of the zeros of the Riemann zeta function in short intervals, Bull. Amer. Math. Soc. 80 (1974), 1339-1342.

2. E. Grosswald, Sur une propriété des racines complexes des fonctions $L(s, X)$, C. R. Acad. Sci. Paris Sér. A-B 263 (1966), A447-A450. MR 34 \#2542.

3. S. Knapowski and $P$. Turán, Comparative prime number theory. I. Introduction, Acta Math. Acad. Sci. Hungar. 13 (1962), 299-314. MR 26 \#3682a.

4. Y. Motohashi, On the distribution of the zeros of Dirichlet's L-functions, Acta Arith. 22 (1972), 107-112. MR 46 \#1727.

5. A. Selberg, Contributions to the theory of Dirichlet's L-functions, Skr. Norske Vid. Akad. Oslo I 1946, no. 3, 62 pp. MR 9, 271.

6. P. Turán, On a problem concerning the zeros of Dirichlet's L-functions, Publ. Ramanujan Inst. 1 (1968/69), 95-100. MR 42 \#1778.

SCHOOL OF MATHEMATICS, INSTITUTE FOR ADVANCED STUDY, PRINCETON, NEW JERSEY 08540

Current address: Department of Mathematics, Rikkyo University, Tokyo, Japan 